\title{
Channel Aging Effects in CoMP Transmission: Gains from Linear Channel Prediction
}

\author{
Lars Thiele, Michael Olbrich, Martin Kurras and Bho Matthiesen \\ Fraunhofer Heinrich Hertz Institute \\ Einsteinufer 37, 10587 Berlin, Germany \\ lars.thiele@hhi.fraunhofer.de
}

\begin{abstract}
CoMP transmission gains attraction for future releases of LTE-Advanced specifications. It is considered for downlink cochannel interference mitigation of OFDMA systems operated at full frequency reuse. However, channel knowledge at the transmitter side will be required, where its usability reduces over time, even under quasi-static mobility. This work studies the range of performance degradation caused by channel aging and delayed utilization for the purpose of joint zero-forcing precoding from a subset of base stations. We demonstrate that channel prediction significantly improves the CoMP performance: There is hardly any difference between ideal and delayed feedback when utilizing prediction filters in low mobility regime.
\end{abstract}

\section{INTRODUCTION}

Cooperative transmission from multiple base stations (BSs) in the multi-cellular system is known to reduce the effects from cochannel interference (CCI). Despite the fact of using zeroforcing $(\mathrm{ZF})$ beamforming, practical systems will always suffer from intra-cluster interference. There is a variety of reasons which destroy the inter-user orthogonality: Channel estimation and quantization, channel aging effects and synchronization errors of multiple BSs. In practical systems, there is always a delay between the channel estimation at the mobile terminal, the feedback of channel quality indicator (CQI) and CSI and the time instant when this estimate is used for composing the following downlink transmission, refer to Fig. 1. As the channel may change during this delay time, the channel observation may be outdated for precoding the next transmission. [1] provides a characterization of the channel evolution due to this aging process for point-to-point MIMO links. However, it assumes equal channel gain for all the links, which is not appropriate for joint transmission (JT) coordinated multi-point (CoMP). In addition, user equipments (UEs) may provide feedback information within a certain interval.

In [2] we focused on synchronization. The contribution of this work is to evaluate the effects caused by channel aging. Therefore, we will describe our system model in sections II and III. Second, we introduce a mismatch between the ideal channel and its estimated or delayed version by evaluating the corresponding relative mean square error (RMSE). Section IV therefore assumes an zero mean additive white Gaussian noise (AWGN) error distribution with different variances $\mu$. In section $\mathrm{V}$, we will study different liner channel prediction methods and their performance in an isolated MIMO link. The overall performance evaluation, including precise modeling of channel time evolutions, is carried out in section VI.

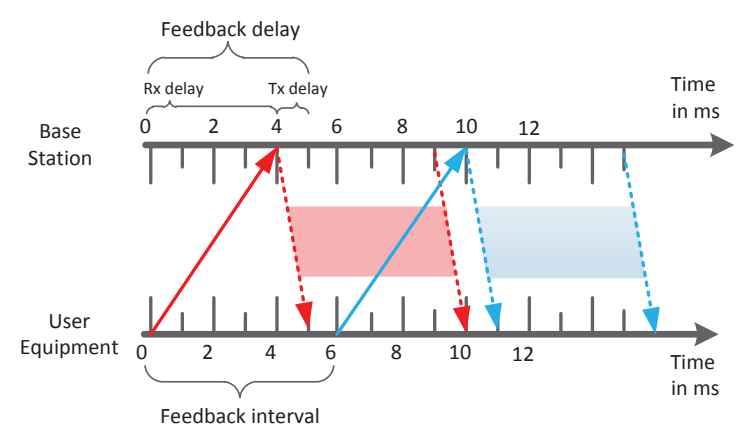

Fig. 1. Definition of feedback delay and feedback interval according to $3 \mathrm{GPP}$ specifications.

\section{Downlink System Model}

By exploiting channel adaptive spatial precoding we can actively reduce the interference inside a cluster of cooperating BSs, denoted as CoMP transmission. We consider a cellular OFDM downlink where a central site is surrounded by multiple tiers of sites. We assume each site to be partitioned into three $120^{\circ}$ sectors, i.e. a set $\mathcal{M}$ consisting of $M=|\mathcal{M}|$ sectors in total. Each sector constitutes a cell, and frequency resources are fully reused in all $M$ cells. $\mathcal{M}_{c}$ represents the set of cells included in a given cluster and $M_{c}=\left|\mathcal{M}_{c}\right|$ denotes its maximum dimension. In order to reduce the overhead for pilots and signalization data, we focus on rather small clusters with up to 3 up to $10 \mathrm{BS}$ sectors. We assume disjoint clusters, i.e. a given $\mathrm{BS}$ cannot belong to more than one cluster operated at the same time/frequency resource. For OFDM systems, the overlap of multiple clusters can be achieved conveniently in the frequency domain. Joint processing is only allowed between BSs belonging to the same cluster, where BSs outside the cluster are not coordinated and thus cause residual intercluster interference. Furthermore, dynamic clustering allows a more efficient power allocation. Mobile users experiencing a weak channel to a given cluster are assigned to another BS cluster [3].

Each cluster selects a set of active users $\mathcal{K}_{c}$ following a specific scheduling metric. In the $c$-th cluster, there are $M_{c}$ BSs, each one equipped with $N_{t}$ transmit antennas, while the $K_{c}=\left|\mathcal{K}_{c}\right|$ users are equipped each with $N_{r}$ receive antennas. The users inside the cluster are served by signals jointly emitted from $M_{c} N_{t}$ transmit antennas, where $M_{c} \cdot N_{t} \geq K_{c} \cdot N_{r}$. 


$$
\begin{aligned}
\mathbf{y}_{k, t}(n)= & \mathbf{H}_{c, k}(n)\left[\mathbf{B}_{c, k}\right]_{:, t}(n-\tau) \sqrt{p_{c, t}} x_{c, t}(n)+\sum_{j \in \mathcal{T}_{s} \backslash\{t\}} \mathbf{H}_{c, k}(n)\left[\mathbf{B}_{c}\right]_{:, j}(n-\tau) \sqrt{p_{c, j}} x_{c, j}(n)+\mathbf{z}_{k}(n) \\
= & \mathbf{H}_{c, k}(n)\left[\left[\mathbf{B}_{c, k}\right]_{:, t}(n)-\left[\boldsymbol{\Delta}_{k}\right]_{:, t}(n, \tau)\right] \sqrt{p_{c, t}} x_{c, t}(n) \\
& +\sum_{j \in \mathcal{T}_{s} \backslash\{t\}} \mathbf{H}_{c, k}(n)\left[\left[\mathbf{B}_{c}\right]_{:, j}(n)-\left[\boldsymbol{\Delta}_{k}\right]_{:, j}(n, \tau)\right] \sqrt{p_{c, j}} x_{c, j}(n)+\mathbf{z}_{k}(n) \\
\mathbf{w}_{k, t}^{H}(n) \mathbf{y}_{k, t}(n) \stackrel{(5)}{=} & \left(\beta_{c, t}-\mathbf{w}_{k, t}^{H}(n) \mathbf{H}_{c, k}(n)\left[\boldsymbol{\Delta}_{k}\right]_{:, t}(n, \tau)\right) \sqrt{p_{c, t}} x_{c, t}(n) \\
& +\sum_{j \in \mathcal{T}_{s} \backslash\{t\}}\left(0-\mathbf{w}_{k, t}^{H}(n) \mathbf{H}_{c, k}(n)\left[\boldsymbol{\Delta}_{k}\right]_{:, j}(n, \tau)\right) \sqrt{p_{c, j}} x_{c, j}(n)+\mathbf{w}_{k, t}^{H}(n) \mathbf{z}_{k}(n) \\
= & \underbrace{\left(\beta_{c, t}-\mathbf{w}_{k, t}^{H}(n) \mathbf{H}_{c, k}(n)\left[\boldsymbol{\Delta}_{k}\right]_{:, t}(n, \tau)\right) \sqrt{p_{c, t}} x_{c, t}(n)}_{\boldsymbol{\vartheta}_{k, t}} \\
& -\underbrace{}_{\sum_{j \in \mathcal{T}_{s} \backslash\{t\}} \mathbf{w}_{k, t}^{H}(n) \mathbf{H}_{c, k}(n)\left[\boldsymbol{\Delta}_{k}\right]_{:, j}(n, \tau) \sqrt{p_{c, j}} x_{c, j}(n)}+\mathbf{w}_{k, t}^{H}(n) \mathbf{z}_{k}(n)
\end{aligned}
$$

The $M_{c} N_{t} \times M N_{t}$ precoding matrix $\mathbf{B}_{c}=\left[\mathbf{B}_{c, 1} \cdots \mathbf{B}_{c, K_{c}}\right]$ contains the precoders $\mathbf{B}_{c, k}$ designed for each of the users. Note, each UE might receive multiple spatial layers at the same time, i.e. $\mathbf{B}_{c, k}$ is of dimension $M_{c} N_{t} \times\left|\mathcal{T}_{s, k}\right|$, where $\mathcal{T}_{s, k}$ denotes the set of spatial layers selected for instantaneous downlink service at user $k$. The maximum number of entries in this set is limited by the number of receive antennas, i.e. $\left|\mathcal{T}_{s, k}\right| \leq N_{r}$. The set $\mathcal{T}_{s}$ combines all selected spatial layers for spatial division multiple access (SDMA) service of the UEs in $\mathcal{K}_{c}$.

For further analysis, we assume the $c$-th cluster is surrounded by $M-M_{c}$ BSs evoking non-coordinated CCI. Thus, the received downlink signal $\mathbf{y}_{k, t}$ for $t \in \mathcal{T}_{s, k} \subset \mathcal{T}_{s}$ at user $k$ in the cellular environment is given by

$$
\begin{aligned}
\mathbf{y}_{k, t}= & \underbrace{\mathbf{H}_{c, k}\left[\mathbf{B}_{c, k}\right]_{:, t} \sqrt{p_{c, t}} x_{c, t}}_{\overline{\mathbf{h}}_{k, t}} \\
+ & \underbrace{\sum_{j \in \mathcal{T}_{s} \backslash\{t\}} \mathbf{H}_{c, k}\left[\mathbf{B}_{c}\right]_{:, j} \sqrt{p_{c, j}} x_{c, j}}_{\boldsymbol{\vartheta}_{k, t}} \\
+ & \underbrace{\sum_{m \in \mathcal{M} \backslash \mathcal{M}_{c}} \sum_{j=1}^{N_{t}} \mathbf{H}_{m, k}\left[\mathbf{B}_{m}\right]_{:, j} \sqrt{p_{m, j}} x_{m, j}+\mathbf{n}}_{\mathbf{z}_{k}}
\end{aligned}
$$

The desired $t$-th data stream $x_{c, t}$ transmitted to the $k$-th user from the $c$-th cluster is distorted by the intra-cluster and inter-cluster interference plus noise aggregated in $\boldsymbol{\vartheta}_{k, t}$ and $\mathbf{z}_{k}$, respectively. $\mathbf{H}_{c, k}$ spans the $N_{r} \times M_{c} N_{t}$ channel matrix for user $k$ formed by the $c$-th cluster and $p_{c, t}$ is its power allocation valid for the $t$-th data stream. Thus, $\boldsymbol{\vartheta}_{k, t}$ denotes the interference generated within the cluster. The $N_{r} \times 1$ vector $\mathbf{n}$ denotes the AWGN samples with covariance $\mathbb{E}\left\{\mathbf{n n}^{H}\right\}=\mathbf{I} \sigma_{n}^{2}$. The noise power consists of the receiver noise figure and the thermal noise power.

The achievable signal-to-interference-and-noise ratio
(SINR) is estimated at each UE, according to

$$
\operatorname{SINR}_{k, t}=\frac{\left|\mathbf{w}_{k, t}^{H} \mathbf{H}_{c, k}\left[\mathbf{B}_{c, k}\right]_{:, t} \sqrt{p_{c, t}}\right|^{2}}{\sum_{j \in \mathcal{\mathcal { T } _ { s } \backslash \{ t \}}}\left|\mathbf{w}_{k, t}^{H} \mathbf{H}_{c, k}\left[\mathbf{B}_{c}\right]_{:, j} \sqrt{p_{c, j}}\right|^{2}+\mathbf{w}_{k, t}^{H}\left[\mathbf{z}_{k} \mathbf{z}_{k}^{H}\right] \mathbf{w}_{k, t}},
$$

with $\mathbf{w}_{k, t}$ being the combining weights at the $k$-th receiver and for data stream $t$.

\section{TIME-VARIANT System Model}

In order to describe the effect of outdated channel knowledge, we extend the above system model description from (3). We define $x_{c, t}(n)$ as the data symbol to be transmitted on the downlink and $\mathbf{H}_{c, k}(n)$ as the MIMO channel matrix at time instance $n .\left[\mathbf{B}_{c, k}\right]_{:, t}(n-\tau)$ denotes the precoding matrix used at time $n$ but based on a $\tau$-old estimate of the channel. Let us assume the precoder to follow a $\mathrm{ZF}$ constraint, which can be obtained using the Moore-Penrose pseudo inverse in case of MISO CSI feedback

$$
\left[\mathbf{B}_{c, k}\right]_{:, t}(n-\tau)=\mathbf{H}_{v i r t}^{H}(n-\tau)\left[\mathbf{H}_{\text {virt }}(n-\tau) \mathbf{H}_{\text {virt }}^{H}(n-\tau)\right]_{(5)}^{-1}
$$

The total transmit power is equally distributed among all active data streams, while we maintain a per antenna power constraint (PAPC) by using a simplified solution from [4]. The transmit power per antenna is chosen according to the row element in $\mathbf{B}_{c}$ with highest norm.

In addition, we may define a difference matrix $\left[\boldsymbol{\Delta}_{k}\right]_{:, t}(n, \tau)$ that relates the $\tau$-old channel inverse with the current precoder as $\left[\mathbf{B}_{c, k}\right]_{:, t}(n)=\left[\mathbf{B}_{c, k}\right]_{:, t}(n-\tau)+\left[\boldsymbol{\Delta}_{k}\right]_{:, t}(n, \tau)$. The received signal $\mathbf{y}_{k, t}(n)$ at the discrete time index $n$ is given by (1) and the equalized signal after ZF precoding simplifies to (2)

From Eq. (2) it becomes obvious that the feedback delay $\tau$ causes a time-variant change in the desired channel vector $\overline{\mathbf{h}}_{k}$ and causes intra-cluster, i.e. inter-user, interference $\boldsymbol{\vartheta}_{k, t}$ depending on the column vectors $\left[\boldsymbol{\Delta}_{k}\right]_{:, j}(n, \tau)$ from matrix $\boldsymbol{\Delta}_{k}(n, \tau)$, as it breaks the inverse relationship between the channel and the precoder. 


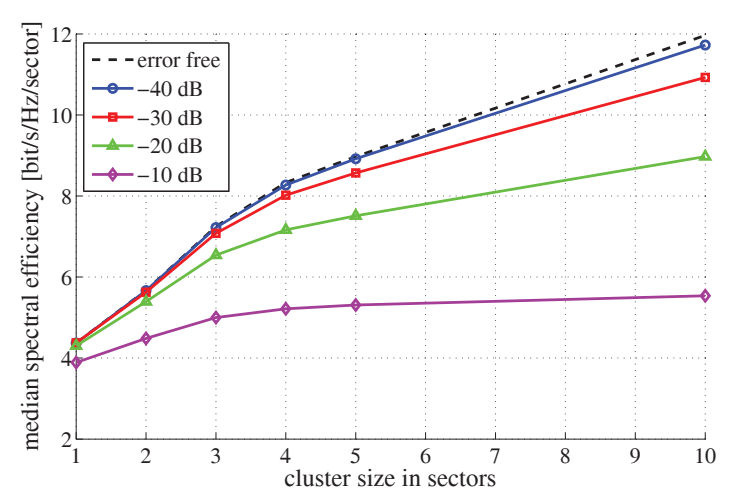

Fig. 2. Performance results as a function of the cluster size $M_{c}$. The normalized MSE is per subchannel, i.e. in case of $M_{c}=10$ sectors in the cluster, each equipped with $N_{t}=2$ antennas, the UE estimates up to $M_{c} \times N_{t}=20$ subchannels with an i.i.d. Gaussian distributed RMSE. Users are equipped with $N_{r}=2$ receive antennas.

\section{MOdELING IMPERFECT CSIT}

In general, we may describe the mismatch between the ideal channel and its estimated or delayed version by evaluating the corresponding RMSE.

$$
\mathrm{rMSE}=\frac{\mathbb{E}|H-\hat{H}|^{2}}{\mathbb{E}|H|^{2}}
$$

To ease comparison of results across different channels, we normalize the mean square error (MSE) by the average channel power. In this following, we evaluate the system level performance of CoMP transmission using multi-user eigenmode transmission (MET) [5], [6] and a dynamic cluster selection and a round-robin scheduling metric for active UEs [7]. Therefore, we assume each UE to decompose its MIMO channel matrix into its dominant eigenspaces, where only the dominant one is used for feedback. In particular, we use CSI feedback from UEs and add a zero mean i.i.d. AWGN term with variance $\mu$, according to (6) per subchannel. This nonperfect CSI is used at the BSs to calculated the $\mathrm{ZF}$ precoding solution.

The erroneous feedback results in a more severe degradation with increasing cluster size. Finally, we increase the cluster size from $M_{c}=1$ up to $M_{c}=10$. Fig. 2 depicts the resulting Shannon information rate per sector as a function of the cluster size $M_{c}$ and accuracy of CSI feedback, i.e. in case of error free and erroneous channel feedback. From this figure it is obvious that an RMSE of $\mu=-10 \mathrm{~dB}$ would restrict the useful cluster size to $M_{c}=3$. In essence, the CoMP gains as function of the cluster size show less saturation behavior for improved multi-cell channel knowledge. Concluding, we observe that the median sector spectral efficiencies are increased by $220 \%$, $300 \%$ and $430 \%$ for coordinating 3, 5 and 10 cells for error free CSI feedback, respectively. These numbers are reduced to $190 \%, 230 \%$ and $300 \%$ in case of erroneous feedback with an MSE of $\mu=-20 \mathrm{~dB}$.

\section{Predicting the Channel Evolution}

In the next section, we want to characterize the range of channel degradation using different linear channel prediction filters. Therefore, let us define $h_{c, k}^{11}$ to be the 1 st sub-channel coefficient of a MIMO channel matrix $\mathbf{H}_{c, k}$. Further assume, $\mathbf{h}_{c, k}^{11}(n)$ being a row vector with the last $p$ observations of the channel frequency response, i.e.,

$$
\mathbf{h}_{c, k}^{11}(n)=\left[h_{c, k}^{11}(n-p+1) \ldots h_{c, k}^{11}(n)\right] .
$$

Then the predicted channel response at time instant $n+\alpha$ is given as

$$
\hat{\mathbf{h}}_{c, k}^{11}(n+\alpha)=\mathbf{h}_{c, k}^{11}(n) \boldsymbol{\omega}_{n}
$$

The optimal weight vector which minimizes the MSE follows from the solution of the normal equations [8], and is given as

$$
\boldsymbol{\omega}_{n}=\left(\mathbf{R}_{h_{c, k}^{11}(n)}+\sigma_{\text {est }}^{2} \mathbf{1}\right)^{-1} \mathbf{r}_{n, \alpha},
$$

with $\sigma_{\text {est }}^{2}$ being the estimation error variance ${ }^{1}$, and

$$
\begin{aligned}
& \mathbf{r}_{n, \alpha}=\left[\begin{array}{lll}
r_{h_{c, k}^{11}(n)}(\alpha+p-1) & \cdots & r_{h_{c, k}^{11}(n)}(\alpha)
\end{array}\right]^{\mathrm{T}}, \\
& \boldsymbol{R}_{h_{i, m}^{11}(n)}=
\end{aligned}
$$

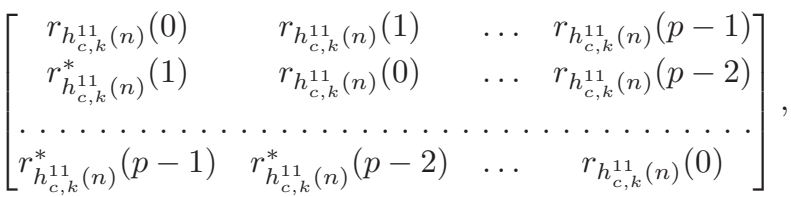

and

$$
\begin{aligned}
r_{h_{c, k}^{11}(n)}(\tau) & =\mathbb{E}_{n}\left\{h_{c, k}^{11}(n+\tau)\left(h_{c, k}^{11}(n)\right)^{*}\right\} \\
& \approx \frac{1}{M} \sum_{n=0}^{M-1-\tau} h_{c, k}^{11}(n+\tau)\left(h_{c, k}^{11}(n)\right)^{*} .
\end{aligned}
$$

In (12) we use the sample autocorrelation function (ACF) [8], [9] to estimate the ACF over the last $M \geq \alpha+p$ samples of the channel response. If not stated different, we use $M=100$ and $p=20$ throughout this work, and solve Eq. (9) at every time instant. Even though the linear system (9) may be solved with computational complexity $O\left(p^{2}\right)$ using the LevinsonDurbin recursion [10], this approach has comparable high computational complexity and memory requirements.

Therefore, we also evaluate well-known adaptive algorithms, namely normalized least-mean-squares (NLMS), and recursive least squares (RLS) [11]. For the latter, we chose the QR-decomposition (QRD) based implementation since it has superior numerical properties in finite precision. Note, that there exist fast RLS algorithms with only $O(p)$ operations per iteration, as opposed to $O\left(p^{2}\right)$ in case of the classical implementation, which may be applied to this problem. Furthermore, we estimate the ACF over the whole channel,

\footnotetext{
${ }^{1}$ We model the estimation error as additive white Gaussian noise with zero mean and variance $\sigma_{\text {est }}^{2}=\frac{\text { SNR }_{\text {est }}}{\mathbb{E}\left\{\left|h_{c, k}^{11}\right|^{2}\right\}}$.
} 
TABLE I

PARAMETER ASSUMPTION FOR CHANNEL PREDICTION.

\begin{tabular}{c|c|c} 
Default value & Description & Affected algorithm \\
\hline$\lambda=0.99$ & Forgetting factor & RLS \\
$\epsilon=0.01$ & Regularization parameter & NLMS \& RLS \\
$M=100$ & Memory length & Wiener \\
$p=20$ & Filter order & all \\
$\alpha=5 \mathrm{~ms}$ & Prediction horizon & all \\
$d=1 \mathrm{~ms}$ & Channel sample spacing & all \\
\hline
\end{tabular}
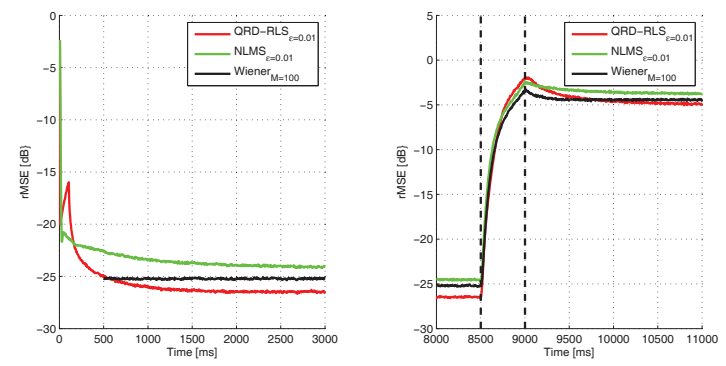

(a) Tracking perf. $3 \mathrm{~km} / \mathrm{h}$

(b) Transition to $50 \mathrm{~km} / \mathrm{h}$

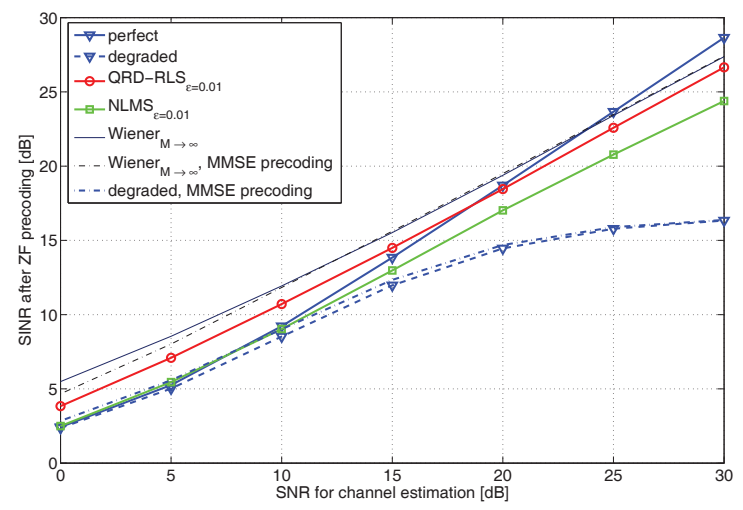

(c) ZF beamforming in $2 \times 2$ MIMO channel

Fig. 3. Channel prediction performance with $p=20$, both feedback delay $\tau$ and interval is set to $5 \mathrm{~ms}$. The channel coefficients are generated using SCME with $3 \mathrm{~km} / \mathrm{h}$ or $50 \mathrm{~km} / \mathrm{h}$ mobility; observation duration is $18^{\prime} 000 \mathrm{~ms}$. In (b), we linearly cross-fade the channels having $3 \mathrm{~km} / \mathrm{h}$ into channels, where UEs move with $50 \mathrm{~km} / \mathrm{h}$, in order to estimate the tracking behavior when channel statistics are changing significantly.

and use (9) to obtain a nearly optimal predictor. This filter will be used to assess the performance of the considered prediction algorithms. Figs. 3(a), 3(b) and 3(c) depict the tracking performance and the performance of $\mathrm{ZF}$ beamforming in a single $2 \times 2 \mathrm{MIMO}$ link. Channel prediction parameters are summarized in Tab. I.

\section{System LeVel Simulation inCluding Channel’s TIME Evolution}

In the following section, we discuss the results we obtained from our system level simulations. Since both, channel prediction and CoMP transmission, strongly depend on the channel modeling, we spend much effort in designing an advanced channel and scenario model for heterogeneous systems. While the core-part based on the recommendation from [12], we
TABLE II

SimULATION ASSUMPTIONS.

\begin{tabular}{l|r} 
Parameter & Value \\
\hline Channel model & According to 3GPP SCME [12] \\
Simulation type & Monte Carlo plus time evolution \\
Drops & 500 \\
Channel evolution & 500 ms with 1 ms resolution \\
Scenario & Urban-macro \\
Propagation & NLOS \\
Large-scale fading & Full buffer \\
Traffic model & $2.6 \mathrm{GHz}$ \\
$f_{c}$ & $3 \mathrm{~km} / \mathrm{h}$ \\
Velocity & 1 \\
Frequency reuse & Geo-correlated parameters maps \\
Signal bandwidth & $18 \mathrm{MHz}, 100 \mathrm{RBs}$ \\
Inter-site distance & $500 \mathrm{~m}$ \\
\hline Number of BSs & 19 having 3 sectors each \\
$N_{t} ;$ spacing & $4 ; 4 \lambda$ \\
Transmit power & $46 \mathrm{dBm}$ \\
Transmit antenna & Azimuth: FWHM of $58^{\circ}$ \\
& Elevation: FWHM of $6.2^{\circ}$ \\
BS height & $10^{\circ}$ electrical downtilt \\
Beamforming & $32 \mathrm{~m}$ \\
Clustering & Cluster-wide MET, ZF constraint \\
User grouping & Network-centric \\
\hline$N_{r} ;$ spacing & 3 user per sector, round-robin \\
UE height & $2 ; \lambda / 2$ \\
Feedback interval & $2 \mathrm{~m}$ \\
Feedback delay & $6 \mathrm{~ms}$ \\
Channel prediction & RLS with $\alpha=\{5,10\}$ ms and $d=1 \mathrm{~ms}$ \\
\hline & $5 \mathrm{~ms}$ \\
\hline
\end{tabular}

have three main extensions included: (a) According to [13], we generate geo-correlated parameter maps for all required large-scale fading coefficients, such as delay spreads, shadow fading, $\mathrm{k}$-factors and angular spreads in azimuth and elevation. (b) We include precise modeling of directional base station antennas with remote electrical tilt (RET) units. Here we use a 3D-model of a Kathrein 80010541 antenna $^{2}$. More details on antenna modeling and its impact on cellular system performance evaluation can be found in [14]. (c) Quasi-deterministic channel's time evolution with drifting of multi-path scattering objects. A detailed list of the multi-cell channel is given in Tab. II.

Each UE is assumed to provide CSI and CQI feedback under the assumption of its own dominant eigenmode vector with respect to the cluster $\mathcal{M}_{c}$. Based on the conveyed downlink user CSI, the cluster determines the sum received power per BS antenna array. For each BS, the scheduler groups the users from $\mathcal{K}_{c}$ according to their individual highest channel gain, yielding $M_{c}$ different user groups $\mathcal{K}_{c, m} \subset \mathcal{K}_{c}$. Within the next step, the round-robin ${ }^{3}$ scheduler entity selects from each user group $\mathcal{K}_{c, m}$ a 3 different users for SDMA service, such that the number of active spatial layers $\mathcal{T}_{s}$ from the cluster $\mathcal{M}_{c}$ is given by $M_{c}\left(N_{t}-1\right)=\left|\mathcal{T}_{s}\right|$. Hence, we reserve one antenna per BS for transmit diversity.

\footnotetext{
${ }^{2}$ http://www.kathrein-scala.com/catalog/80010541.pdf

${ }^{3}$ Note, this very simple metric ensures a certain degree of fairness between the users.
} 


\section{CONCLUSION}

Fig. 4 shows the results, obtained from our computer simulations. In particular, Fig. 4(a) depicts the median RMSE at different time instances, i.e. after certain settling times of the prediction filter. The error starts at a relatively low value, then starts rising since the RLS, at this stage, covered insufficient channel statistics while the probability for a changing channel is increasing. After a certain settling time, channel statistics gets more sufficient and thus the error starts dropping and converges to a minimum value. Note, for the selected time window of $500 \mathrm{~ms}$, we cannot observe this convergence behavior.

Figs. 4(b) and 4(c) provide performance results for cluster size $M_{c}=1$ and $M_{c}=3$, respectively. The ideal case depicts the system with perfect, non-delayed channel knowledge, while the other CDFs show the performance with delay and with or without prediction filter: At cluster size $M_{c}=1$, we hardly observe any degradation without prediction for $5 \mathrm{~ms}$ feedback delay and only small loss when experiencing $10 \mathrm{~ms}$ delay. For $M_{c}=3$ and $10 \mathrm{~ms}$ delay, the degraded system suffers from $8 \%$ loss. Note, from Fig.2, we would expect a stronger degradation, which is attributed to the modeling of the error distribution. As a result, we conclude that the errors from channel outdating may not follow a Gaussian distribution, which was assumed in section IV. When we apply the RLS channel prediction, there is a hardly any loss at $10 \mathrm{~ms}$ delay and $3 \mathrm{~km} / \mathrm{h}$ for both cluster sizes.

\section{ACKNOWLEDGEMENTS}

The authors are grateful for stimulating discussions with partners from the Celtic WINNER+ consortium as well as Konstantinos Manolakis and Sławomir Stańczak.

\section{REFERENCES}

[1] V. Pohl, P. Nguyen, V. Jungnickel, and C. von Helmolt, "Limits of the Achievable Symbol Rate in Flat Fading MIMO Systems," in Proc. 14th IEEE Intern. Symposium on Personal, Indoor and Mobile Radio Commun. (PIMRC), vol. 3, Bejing, China, Sep. 2003, pp. 2548-2552.

[2] K. Manolakis, L. Thiele, C. Oberli, T. Haustein, and V. Jungnickel, "Impairment modeling for joint transmission CoMP," in IEEE 2nd International Conference on Wireless Communications, Vehicular Technology, Information Theory and Aerospace \& Electronic System Technology (Wireless VITAE), Chennai, India, Mar. 2011, invited.

[3] F. Boccardi, H. Huang, and A. Alexiou, "Network mimo with reduced backhaul requirements by mac coordination," in Signals, Systems and Computers, 2008 42nd Asilomar Conference on, Oct. 2008, pp. 11251129.

[4] H. Zhang and H. Dai, "Cochannel interference mitigation and cooperative processing in downlink multicell multiuser MIMO networks," Eurasip Journal on Wireless Communications and Networking, vol. 2004, no. 2, pp. 222-235, 2004.

[5] F. Boccardi and H. Huang, "A near-optimum technique using linear precoding for the MIMO broadcast channel," Acoustics, Speech and Signal Processing, 2007. ICASSP 2007. IEEE International Conference on, vol. 3, pp. III-17-III-20, April 2007.

[6] M. Noda, M. Muraguchi, T. G. Khanh, K. Sakaguchi, and K. Araki, "Eigenmode tomlinson-harashima precoding for multi-antenna multiuser mimo broadcast channel," in Information, Communications Signal Processing, 2007 6th International Conference on, 10-13 2007, pp. 1 -5 .

[7] L. Thiele, V. Jungnickel, and T. Haustein, "Interference management for future cellular ofdma systems using coordinated multi-point transmission," IEICE Transactions on Communications, vol. E93.B, no. 12, pp. 3228-3237, Dec. 2010, invited.

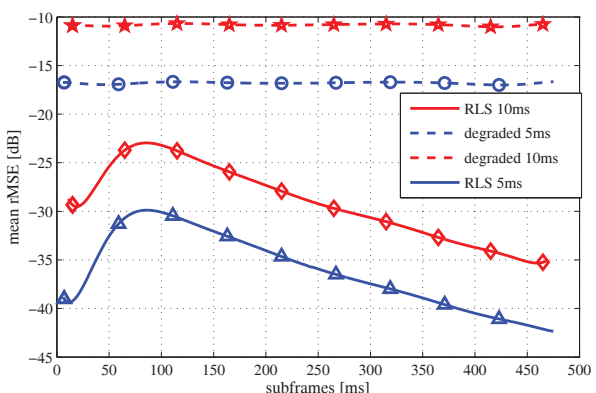

(a) RMSE

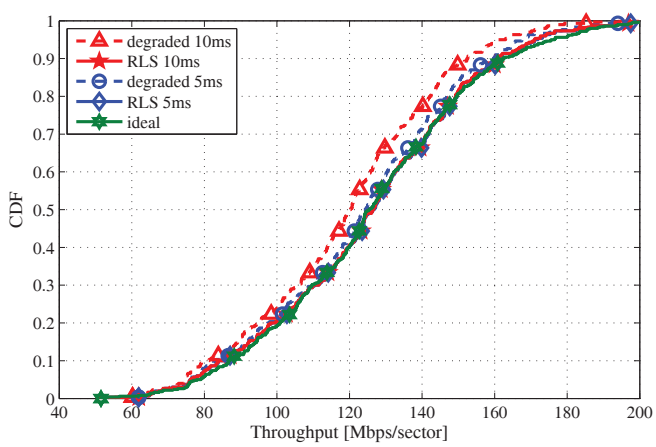

(b) Cluster size $M_{c}=1$, single-cell ZF precoding.

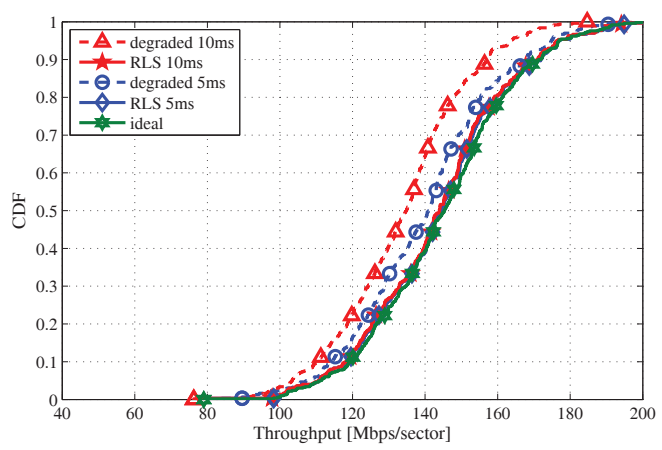

(c) Cluster size $M_{c}=3$, i.e. intra-site CoMP.

Fig. 4. Overall simulation results taking precise channel evolution into account and specific channel feedback interval.

[8] I. C. Wong, A. Forenza, R. W. Heath, and B. L. Evans, "Long range channel prediction for adaptive OFDM systems," in Proc. Conf Signals, Systems and Computers Record of the Thirty-Eighth Asilomar Conf, vol. 1, 2004, pp. 732-736.

[9] S. J. Orfanidis, Optimum Signal Processing, 2nd ed. McGraw-Hill, 1988. [Online]. Available: http://www.ece.rutgers.edu/ orfanidi/osp2e/

[10] G. H. Golub and C. F. Van Loan, Matrix Computations, 3rd ed. John Hopkins University Press, 1996.

[11] A. H. Sayed, Adaptive Filters. Wiley-Interscience, 2008.

[12] 3GPP TR 25.996 V10.0.0, "Spatial channel model for multiple input multiple output (MIMO) simulations (release 10)," mar 2011. [Online]. Available: http://www.tkk.fi/Units/Radio/scm/

[13] H. Claussen, "Efficient modelling of channel maps with correlated shadow fading in mobile radio systems," in Personal, Indoor and Mobile Radio Communications, 2005. PIMRC 2005. IEEE 16th International Symposium on, vol. 1, sept. 2005, pp. $512-516$.

[14] L. Thiele, T. Wirth, K. Börner, M. Olbrich, V. Jungnickel, J. Rumold, and S. Fritze, "Modeling of 3D Field Patterns of Downtilted Antennas and Their Impact on Cellular Systems," International ITG Workshop on Smart Antennas (WSA 2009), Feb. 2009. 